\title{
Anastomosis Is Possible with an Acceptable Low Rate of Complications Compared to a Diverting Stoma in Surgery for Ovarian Cancer
}

\author{
Vibe Munk Bertelsen ${ }^{1}$, Gitte Ørtoft Lykkegård ${ }^{2}$, Lone Kjeld Petersen ${ }^{3 \text {, * }}$ \\ ${ }^{1}$ Department of Gynecology and Obstetrics, Aarhus University Hospital, Aarhus, Denmark \\ ${ }^{2}$ Department of Gynecology and Obstetrics, Copenhagen University Hospital, Copenhagen, Denmark \\ ${ }^{3}$ Department of Gynecology and Obstetrics, Odense University Hospital, Odense, Denmark

\section{Email address:} \\ vibe_bertelsen@hotmail.com (V.M. Bertelsen), ortoft@dadlnet.dk (G. Ø. Lykkegård), lone.kjeld.petersen@gmail.com (L. K. Petersen) \\ ${ }^{*}$ Corresponding author
}

\section{To cite this article:}

Vibe Munk Bertelsen, Gitte Ørtoft Lykkegård, Lone Kjeld Petersen. Anastomosis Is Possible with an Acceptable Low Rate of Complications Compared to a Diverting Stoma in Surgery for Ovarian Cancer. Journal of Cancer Treatment and Research. Vol. 7, No. 2, 2019 , pp. 28-32. doi: $10.11648 /$ j.jctr.20190702.11

Received: March 10, 2019; Accepted: April 25, 2019; Published: June 5, 2019

\begin{abstract}
Radical surgery for advanced stage of ovarian cancer may lead to bowel resection and consequently either an anastomosis or a diverting stoma. This study investigates whether it is possible to find selection criteria which predict benefits from an anastomosis compared to a diverting stoma, in order to prevent complications and leakage. Consecutive patients with ovarian/tuba/peritoneal cancer undergoing initial bowel resection at Aarhus University Hospital, Denmark, between March 2012 and December 2015 were retrospectively identified. Among 67 patients with bowel resections, 32 patients had a stoma and 35 patients had an anastomosis. No significant differences were observed in the two groups regarding age, BMI, smoking, ASA classification, FIGO stage, plasma albumin, the ability to undergo radical surgery, or time to initiate chemotherapy. The length of hospital stay was longer for patients with a stoma $(\mathrm{P}=0.01)$. An anastomotic leakage lead to reoperation for $8.6 \%$ of the anastomosis patients. Patients who were reoperated due to leakage, initiated chemotherapy after 21-45 days. Only smoking was identified as a preoperative risk factor for leakage after bowel anastomosis in relation to debulking surgery for ovarian cancer. The complication rate among patients with an anastomosis was acceptably low, and the time from surgery to start of chemotherapy was the same as in patients with a stoma. This study supports the hypothesis that an anastomosis can be safely performed in patients with advanced ovarian cancer.
\end{abstract}

Keywords: Ovarian Cancer, Bowel Resection, Anastomotic Leakage, Stoma, Selection Criteria

\section{Introduction}

Ovarian cancer accounts for $3 \%$ of all cancers within the Nordic countries, and approximately 550 new cases are diagnosed in Denmark every year. It is the most lethal gynecological cancer, and the treatment outcome is generally poor, with a relative 1 -year and 5-year survival rates of $78 \%$ and $40 \%$ [1]. The cancer is predominantly asymptomatic in its early stages, and up to $70 \%$ of women with ovarian cancer are diagnosed at FIGO stage III-IV, when the cancer has spread outside the pelvis. [2].

Combined treatment consisting of surgery and chemotherapy is the standard of care in ovarian cancer patients [3]. The surgery has two purposes: (1) to ensure proper cancer staging, and (2) the removal of all tumor, if possible, to improve the outcome of chemotherapy [4]. A complication-free postoperative period has high priority in order to ensure that the patients can receive the necessary adjuvant chemotherapy. Complete resection of tumor in patients with advanced disease often includes bowel resection [5]. Even though patients may benefit from avoiding a stoma, an anastomosis involves the risk of 
leakage, reoperation and prolonged recovery [6]. Breakdown of an anastomosis and the resulting leakage is one of the most feared complications after bowel resection because it is associated with considerable morbidity and increased mortality. [7].

No randomized studies have compared the efficacy of the two surgical procedures, and no selection criteria have been identified to predict which patients could benefit from an anastomosis compared to a diverting stoma. At many institutions, the decision to do either a bowel resection with anastomosis or a colostomy during cytoreductive surgeries, is therefore taken intraoperatively by personal choice from the operating surgeon. Choice of treatment may rely both on surgical considerations and the patient's health status and risk factors.

The aim of this study was to identify possible selection criteria that can be used as significant predictors in choosing optimally between performing either a stoma or an anastomosis, and to predict the risk of leakage.

\section{Materials and Methods}

\subsection{Design and Study Sample}

Through the Danish National Patient Registry, all consecutive patients who underwent bowel resection for primary or recurrent ovarian/tubal/peritoneal cancer were identified retrospectively from March 1, 2012 to December 31, 2015 at Aarhus University Hospital, Aarhus, Denmark. The ICD-10 code was used for ovarian cancer (DC569) in combination with procedures on the bowel: KJFA or KJFB for bowel resection, KJFF or KJFH for total colectomy, KJFW96 for operation on small or large bowel, KJGB10 for resection of rectum with colostomy.

Seventy-seven patients met that criteria. According to the patient files, eight patients had neither a stoma or an anastomosis, and two did not have ovarian cancer. The study population therefore consisted of 67 patients.

\subsection{Materials}

Data were collected by reviewing the electronic patient files with regard to BMI, smoking habits, ASA classification, and plasma albumin within the last 14 days before surgery. Age was calculated on the day of surgery. Follow-up information included length of hospital stay (LOS), mortality within 30 or 90 days of surgery, and time between surgery and the initiation of chemotherapy.

Information about stoma, anastomosis, type of bowel resection, use of drainage, leak-rate, how leakage was diagnosed, post-operative complications, and re-surgeries were based on the surgeon's or nurse's case notes. Macroscopic complete resection (complete cytoreduction $(\mathrm{CC})=0$ ) was defined as no visible residual tumor, and no carcinosis according to the surgeons.

Leakages were diagnosed by careful postoperative surveillance including scoring of patients (temperature, pulse, respiration, blood pressure) every eight hours for the first three postoperative days. Moreover, WBC and CRP were analysed every 24 hours, and CT scans were performed whenever a leakage could not be ruled out based on clinical signs or abnormal laboratory tests.

All patients were staged according to the FIGO 2009 system based on data from the medical record or the National Pathology Data Bank.

\subsection{Statistical Methods}

All data were analyzed using the program Stata/IC 13.1. To compare and test for differences in medians, the Wilcoxon rank-sum test was used. Five patients who never started chemotherapy after the primary surgery were excluded from the calculations regarding "time to initiation of chemotherapy".

Differences in binary variables were found using a twosample test of proportions. To detect linear relationships between variables, Pearson's correlation coefficient was applied. For all tests, the alpha level was set to $5 \%$ for significant results.

Permission was given by the Danish Data Protection Agency (J-nr.:2014-41-3431) to conduct the study. The National Board of Health has authorized the publication (case number: 3-3013-866/2/).

\section{Results}

\subsection{Patient Characteristics}

Sixty-seven patients were included in the study. Nine patients (13\%) had both a stoma and an anastomosis performed at the primary surgery, who were included in the stoma group. In total, 32 patients $(48 \%)$ had either a stoma or a stoma with an anastomosis, and 35 patients (52\%) had only an anastomosis. Age, BMI, smoking, ASA classification, preoperative albumin, and operation due to recurrent disease were comparable between the groups (Table 1). The majority of patients were diagnosed with advanced stage disease as $90 \%$ had stage IIIC-IV ovarian cancer. One patient with FIGO stage IA disease had an anastomosis due to a surgical complication with perforation of the bowel during laparoscopic staging. FIGO stage for one patient was not found.

The shortest time to chemotherapy was 15 days, whereas the longest period was 61 days. Five patients never started chemotherapy after surgery because of their clinical conditions, not because of surgery complications. Four of these patients had a stoma. 
Table 1. Patient characteristics.

\begin{tabular}{llll}
\hline & Stoma $\mathbf{n}=\mathbf{3 2}$ & Anastomosis n $=35$ & P \\
\hline Length of hospitalization* & $12(4-34)$ & $9(4-34)$ & $0.01 \mathrm{~S}$ \\
Age* $_{\text {BMI* }}$ & $66.5(40-80)$ & $66(45-79)$ & $0.39 \mathrm{NS}$ \\
Smoking** & $24.3(15.6-35.4)$ & $23(16.4-39.4)$ & $0.48 \mathrm{NS}$ \\
ASA classification** & 5 & 5 & $0.92 \mathrm{NS}$ \\
I & 6 & 7 & $0.90 \mathrm{NS}$ \\
II & 18 & 19 & $0.87 \mathrm{NS}$ \\
III & 8 & 9 & $0.95 \mathrm{NS}$ \\
Albumin* & $33(20-45)$ & $35(22-45)$ & $0.61 \mathrm{NS}$ \\
Initiation chemotheraphy (days)* & $29(15-61)$ & $28(15-48)$ & $0.77 \mathrm{NS}$ \\
FIGO stage* & & 4 & $0.80 \mathrm{NS}$ \\
I- IIIb & 3 & 30 & $0.54 \mathrm{NS}$ \\
IIIc - IV & 29 & 3 & $0.35 \mathrm{NS}$ \\
Recurrent ovarian cancer** & 1 & & \\
\hline
\end{tabular}

$\mathrm{S}$ indicates significant at 5\% level, NS indicates non-significant at 5\% level. P-values were produced using Wilcoxon rank-sum test or a two-sample test of proportions. Median values are marked with *, and count variables are marked with **.

\subsection{Surgical Procedures}

Standard surgery included bilateral salpingooophorectomia, hysterectomy, and removal of the omentum, appendix and pelvic, and paraaortic lymph nodes. During surgery, the spleen was removed in $22.4 \%$ and partial peritonectomy was performed in $61.2 \%$ of the cases.

The vast majority of patients $(90 \%)$ had large bowel resections (Table 2). Small intestine and colon resection were significantly associated to performance of anastomosis, while stoma was more often associated to resection of the colon and rectum $(\mathrm{P}=0.01)$. Three patients had a stoma with no bowel resections in order to relieve a bowel obstruction. Macroscopic complete resection did not differ significantly between the two groups as $\mathrm{CC}=0$ was achieved in $77 \%$ of the stoma patients and in $89 \%$ of the anastomosis patients. Four patients $(6 \%)$ had two anastomoses during surgery: Two patients in the stoma group, and two in the anastomosis group.

Table 2. Bowel resection in patients operated for Ovarian Cancer.

\begin{tabular}{llll}
\hline & Stoma $\mathbf{n}=\mathbf{3 2}$ & Anastomosis n $=\mathbf{3 5}$ & $\mathbf{P}$ \\
\hline $\begin{array}{l}\text { Standard surgery** } \\
\text { Bowel resection** }\end{array}$ & 31 & 35 & $0.29 \mathrm{NS}$ \\
Small intestine & $0(0.0 \%)$ & $7(20.0 \%)$ & $0.01 \mathrm{~S}$ \\
Small intestine + Colon & $3(10.3 \%)$ & $2(5.7 \%)$ & $0.57 \mathrm{NS}$ \\
Small intestine + Colon + Rectum & $1(2.9 \%)$ & $1(3.5 \%)$ & $0.95 \mathrm{NS}$ \\
Colon & $12(41.4 \%)$ & $22(62.9 \%)$ & $0.04 \mathrm{~S}$ \\
Colon + Rectum & $11(37.9 \%)$ & $3(8.6 \%)$ & $0.01 \mathrm{~S}$ \\
Rectum & $2(6.9 \%)$ & $0(0.0 \%)$ & $0.13 \mathrm{NS}$ \\
Macroscopic complete resection** & $24(75 \%)$ & $31(89 \%)$ & $0.15 \mathrm{NS}$ \\
\hline
\end{tabular}

$\mathrm{S}$ indicates significant at $5 \%$ level, NS indicates non-significant at $5 \%$ level. P-values were produced using Wilcoxon rank-sum test or a two-sample test of proportions. Median values are marked with *, and count variables are marked with **.

\subsection{Postsurgical Complications and Mortality Rate}

Postsurgical complications are given in Table 3.

In all, $8.6 \%$ of the patients experienced anastomotic leakage. If those with only a small intestine resection and an anastomosis are excluded, the leakage rate for anastomosis was $10.7 \%$. Four patients had re-surgery, three due to anastomotic leakage, and one due to perforation of the transverse colon. They all received an anastomosis at the primary surgery, and all were managed with colostomy at reoperation. All four cases were confirmed by CT scans, ordered due to clinical conditions. In one case chemotherapy was never started, and this patient died 84 days after primary surgery of a non-surgical related condition. The other three patients started chemotherapy after 21,40 , and 45 days (median value 28 days), and survived more than 90 days.

Tabel 3. Postoperative complications in patients operated for Ovarian Cancer.

\begin{tabular}{llll}
\hline & Stoma $\mathbf{n}=\mathbf{3 2}$ & Anastomosis $\mathbf{n}=\mathbf{3 5}$ & \multicolumn{1}{c}{ P } \\
\hline Leakage** & 0 & 3 & $0.09 \mathrm{NS}$ \\
Re-operation** & 0 & 4 & $0.05 \mathrm{~S}$ \\
30 days mortality** & 0 & 0 & NS \\
90 days mortality** & 5 & 1 & 0.07 NS \\
\hline
\end{tabular}

$\mathrm{S}$ indicates significant at 5\% level, NS indicates non-significant at 5\% level. P-values were produced using Wilcoxon rank-sum test or a two-sample test of proportions. Median values are marked with *, and count variables are marked with **. 


\subsection{Six Deaths}

Overall, six patients (9\%) died within 90 days after surgery, none within 30 days. Three patients had macroscopic complete resection, and in three patients the operation was performed in order to relieve a bowel obstruction due to an extensive tumor spread. None of the deaths were related to surgery complications or to anastomotic leak. Two died later in hospital caused by pneumonia, fever and sepsis. The other four died from progression of cancer.

\subsection{Correlations}

The macroscopic complete resection was correlated with a higher BMI, and less risk of death within 90 days. Risk of death within 90 days was correlated with stoma, longer LOS, and low albumin before surgery. A reoperation and the risk of leakage were both significantly correlated with smoking and inversely correlated with stoma. The time to initiation of chemotherapy seemed to be shorter for anastomosis patients, and longer with leakage, LOS, and high BMI.

\section{Discussion}

The prognostic benefits of complete cytoreduction during surgery for advanced ovarian cancer is well established. Radical debulking surgery often requires bowel resection, but the consequences of performing a bowel resection with a stoma compared to an anastomosis, have not been extensively investigated. A primary anastomosis, especially in the rectum with the risk of subsequent leakage, may postpone further treatment with chemotherapy. The data in this study do not support a specific uniform strategy for choosing either stoma or anastomosis during surgery for advanced ovarian cancer, in order to prevent complications and leakage. The present result showed no differences between the groups regarding preoperative factors like age, ASA score, BMI, FIGO stage or albumin concentrations. Another retrospective study indicates that low anterior resection with anastomosis may be an optimal bowel surgery procedure. When this procedure was compared with resection of the rectosigmoid colon and formation of a stoma, no differences in outcomes, postoperative complications, or survival were found [8].

Among patients in this study with an anastomosis, only smoking could be identified as a risk factor for leakage. Factors like age, comorbidity and frailty as evaluated by ASA score, BMI, and albumin level were also not associated with an increased risk of leakage. Grimm et al. [9] report similar observations, as they could not identify pre- or intraoperative risk factors for leakage of bowel anastomosis, performed in relation to debulking surgery for ovarian cancer. The results in this study are also in agreement with those reported by Bartl et al. [10], and indicate that bowel anastomosis may be performed with acceptable leakage rate even in elderly patients, and patients with comorbidity. In case of a leakage, early diagnosis and reoperation is important to minimize the consequences of the complications. Based on the routine surveillance in this study, all the patients with a leakage (except for one), had acceptable delay in time to adjuvant chemotherapy which was initiated within 21-45 days after the primary surgery. The data showed a delay in time to chemotherapy within the same range as reported by Bartl et al. [10]. The patients in this study had reoperation within 7-11 days after the primary surgery, whereas Bartl et al. [10] had 217 days to revision. Unlike Bartl et al. [10] there exists a standardized follow-up regime, as described above, with close post-operative surveillance. This to ensure diagnostics of leakage as soon as possible.

In the literature, anastomotic leak rates range from $0.8 \%$ to $3.2 \%$ in surgery for gynecologic malignancy. The subsequent mortality rate after an anastomotic leakage after rectosigmoid resection is $7.3 \%$ to $16 \%[10,11]$. This study found a 90-day mortality in less than $3 \%$ of patients with anastomosis, which is within the reported results by others. Especially resection of the rectum implies risk of a subsequent leakage. The leakage rate in this study consisting of a group of patients who all had bowel resections is acceptable $(8.6 \%)$, especially when it is considered that complete cytoreduction was obtained in $82 \%$ of the patients. The study period represents the initial phase of a more extensive surgical approach in advanced ovarian cancer treatment at Aarhus University Hospital. The expectance is a decrease in the leakage rate when the procedures become routine among the surgeons involved.

Low plasma albumin concentrations are associated with an increased risk of anastomosis leakage [11]. In this study plasma albumin was significantly correlated with the risk of death within 90 days, but not with the risk of leakage. Bartl et al. [10] neither found albumin, or any other predictive risk factors for leakages.

The strength of this study is the completeness of data due to the Danish personal code number that makes it possible to trace patients across all databases in the health care system. Moreover, the consecutive inclusion of all patients with bowel resection secures the description of the entire group of patients and prevents selection bias. Consequently, the study group reflects the clinical setting in a tertiary centre and the results may be of general interest.

A limitation of this study is the small sample size which have an impact on the statistical power. Moreover, the surgeon's experience and the clinical setting has without doubt influenced the decision as to perform an anastomosis or a stoma during the operation. The increased 90 days mortality rate in patients treated by a stoma and prolonged hospital stay may indicate more advanced disease in these patients, where surgery may have had aims of palliative care. The number of patients who did not initiate chemotherapy in the group of patients with stoma supports this observation. Significantly more stoma patients had resection of the colon and rectum. Possibly, a stoma was chosen for these patients to secure less risks of leakages and complications.

Studies in patients with ovarian cancer that compare 
surgical procedures, anastomosis vs. stoma, are sparse. In rectal cancer patients, a protective stoma may decrease the incidences of an anastomotic leakage and a reoperation [12]. The leakage rate in low rectum anastomoses have been reported up to $10 \%[7,13]$, but a direct comparison between ovarian cancer and rectal cancer patients can, however, not be justified due to tumor biology and the anatomic location of tumor. A rectal cancer surgery often implies a low rectal resection to obtain free margins, whereas a rectal resection in ovarian cancer is frequently performed to remove the tumor in the pouch of Douglas next to the upper part of the rectum and lower part of the sigmoid colon. The higher anastomosis in ovarian cancer patients may be associated with a lower risk of a subsequent anastomotic leakage.

Although a protective stoma did not impact surgical outcomes in our and previous studies, about half of the surgeries for ovarian cancer, including bowel resection, at Aarhus University Hospital involve a stoma. The interdisciplinary team work where general surgeons assist in many advanced ovarian cancer operations may influence the decision regarding when to use a stoma instead of, or combined with, a bowel anastomosis. The recommendation to construct a protective stoma in rectal cancer patients, who undergo low rectal resection, is based on an anastomotic leak rate described in a meta-analysis ranging from $1-24 \%$ in colorectal patients [12]. With regard to surgical collaboration, it is important to recognize the different biological and anatomic locations of the various types of pelvic tumors. The differences in surgical approaches emphasize the importance of collaboration in the surgical treatment of advanced ovarian cancer patients, and the presence of surgeons specially trained in gynaecologic oncology is mandatory during these operations.

\section{Conclusion}

In this small study in advanced ovarian cancer patients, no selections criteria were found to significantly predict benefits from an anastomosis compared to a stoma, except for smoking which was significantly correlated with a resulting leakage. The leakage rate $(8.6 \%)$ and 90 -day mortality rate $(2.9 \%)$ after anastomosis were acceptable. The results indicate that resection of the bowel with an anastomosis can be safely performed at cytoreductive surgery in ovarian cancer patients. Early detection of a leakage may prevent unnecessary delay in a subsequent adjuvant chemotherapy. The results need confirmation in a larger study population, and additional studies are needed to clarify the risks of leakages in order to create an appropriate guide for intraoperative surgical decisions.

\section{References}

[1] NORDCAN (Nordic Cancer database program). Updated December 2017. http://wwwdep.iarc.fr/NORDCAN/English/frame.asp
[2] DGCD annual Report http://www.dgcg.dk/index.php/arsrapport

[3] Kirsten Jochumsen K., Larsen S., and Neumann G. November 2016. https://www.sundhed.dk/sundhedsfaglig/information-tilpraksis/syddanmark/almen-praksis/patientforloeb/icpcoversigt/x-kvindelige-koensorganger-inklmammae/ovariecancer/

[4] Vitale SG, Marilli I, Lodato M, and Tropea A, and Cianci A. "The role of cytoreductive surgery in advanced-stage ovarian cancer: a systematic review." Updates Surg. 2013 Dec; 65(4):265-70. doi: 10.1007/s13304-013-0213-4. Epub 2013 May 8

[5] Schorge J. O., McCann C., and Del Carmen MG. "Surgical Debulking of Ovarian Cancer: What Difference Does It Make?". Rev Obstet Gynecol. 2010 Summer;3(3):111-7

[6] O'Leary D. P., Fide C. J., Foy C, and Lucarotti M. E. "Quality of life after low anterior resection with total mesorectal excision and temporary loopileostomy for rectal carcinoma". Br J Sung. 2001 Sep;88(9):1216-20

[7] Mourton S. M., Temple L. K., Abu-Rustum N. R., Gemignani M. L., Sonoda Y., Bochner B. H, et al. "Morbidity of rectosigmoid resection and primary anastomosis in patients undergoing primary cytoreductive surgery for advanced epithelial ovarian cancer". Gynecol Oncol. 2005 Dec; 99(3):608-14. Epub 2005 Sep 8

[8] Kim H. S., Kim E. N., and Jeong S. Y. "Comparison of the efficacy of low anterior resection with primary anastomosis and Hartmann's procedure in advanced primary or recurrent epithelial ovarian cancer". Eur J Obstet Gynecol Reprod Biol. 2011 Jun; 156(2):194-8. doi: 10.1016/j.ejogrb.2011.01.003. Epub 2011 Feb 2

[9] Grimm C., Harter P., Alesina P. F., Prader S., Schneider S., Ataseven B., et al.. "The impact of type and number of bowel resections on anastomotic leakage risk in advanced ovarian cancer surgery". Gynecol Oncol. 2017 Sep; 146(3):498-503. doi: 10.1016/j.ygyno.2017.06.007. Epub 2017 Jun 10

[10] Bartl T., Schwameis R., Stift A., Bachleitner-Hofmann T., Reinthaller A., Grimm C., et al. "Predictive and Prognostic Implication of Bowel Resections During Primary Cytoreductive Surgery in advanced Epithelial Ovarian Cancer". Int J Gynecol Cancer. 2018 Nov; 28(9):1664-1671. doi: 10.1097/IGC.0000000000001369

[11] Richardson D. L., Marian A., and Cliby W. A. "Risk factors for anastomotic leak after recto-sigmoid resection for ovarian cancer". Gynecol. Oncol. 2006 Nov; 103(2):667-72. Epub 2006 Jun 23

[12] Wu S. W., Ma C. C., and Yang Y. "Role of protective stoma in low anterior resection for rectal cancer: A meta-analysis". World J Gastroenterol. 2014 Dec 21; 20(47):18031-7. doi: 10.3748/wjg.v20.i47.18031

[13] Schmidt O, Merkel S, and Hohenberger W. "Anastomotic leakage after low rectal stapler anastomosis: significance of intraoperative anastomotic testing”. Eur J Surg Oncol 2003 Apr;29(3):239-43 